\title{
Dominant protein interactions that influence the pathogenesis of conformational diseases
}

\author{
Jordan Wright, ${ }^{1}$ Xiaofan Wang,, ${ }^{1,2}$ Leena Haataja, ${ }^{1}$ Aaron P. Kellogg, ${ }^{1}$ \\ Jaemin Lee, ${ }^{1}$ Ming Liu, ${ }^{1}$ and Peter Arvan'
}

\begin{abstract}
1Division of Metabolism, Endocrinology and Diabetes, University of Michigan Medical Center, Ann Arbor, Michigan, USA. ${ }^{2}$ Department of Pharmacology and Experimental Therapeutics, Boston University School of Medicine, Boston, Massachusetts, USA.
\end{abstract}

\begin{abstract}
Misfolding of exportable proteins can trigger endocrinopathies. For example, misfolding of insulin can result in autosomal dominant mutant INS gene-induced diabetes of youth, and misfolding of thyroglobulin can result in autosomal recessive congenital hypothyroidism with deficient thyroglobulin. Both proinsulin and thyroglobulin normally form homodimers; the mutant versions of both proteins misfold in the ER, triggering ER stress, and, in both cases, heterozygosity creates potential for cross-dimerization between mutant and WT gene products. Here, we investigated these two ER-retained mutant secretory proteins and the selectivity of their interactions with their respective WT counterparts. In both cases and in animal models of these diseases, we found that conditions favoring an increased stoichiometry of mutant gene product dominantly inhibited export of the WT partner, while increased relative level of the WT gene product helped to rescue secretion of the mutant partner. Surprisingly, the bidirectional consequences of secretory blockade and rescue occur simultaneously in the same cells. Thus, in the context of heterozygosity, expression level and stability of WT subunits may be a critical factor influencing the effect of protein misfolding on clinical phenotype. These results offer new insight into dominant as well as recessive inheritance of conformational diseases and offer opportunities for the development of new therapies.
\end{abstract}

\section{Introduction}

Several human "conformational diseases" of the secretory pathway are caused by mutations in exportable proteins blocking their export from the ER (1). Loss of function in post-ER compartments is often observed as autosomal recessive disease. By contrast, other disorders caused by gain-of-toxic-function mutations can ultimately lead to cell death that may trigger autosomal dominant disease (2).

Given that both autosomal dominant and recessive mutations can be found in exportable proteins that form homodimers and the much higher frequency of heterozygosity than homozygosity in the global population, it is critical to understand how cross-dimerization between WT and mutant gene products might influence clinical phenotypes. To study this, we have examined mutant forms of proinsulin (linked to autosomal dominant disease) and thyroglobulin ( $\mathrm{Tg}$ ) (linked to autosomal recessive disease).

Proinsulin, the major protein synthesized by pancreatic $\beta$ cells, is cotranslationally translocated into the ER. In the ER, proinsulin is thought to form noncovalent homodimers that proceed in the distal secretory pathway to form homohexamers that undergo endoproteolytic processing to mature insulin and C-peptide (3). Recently, 26 distinct coding sequence mutations in proinsulin have been found responsible for a gain of toxic function underlying the autosomal dominant syndrome of mutant INS gene-induced diabetes of youth (MIDY), in which secretion of coexpressed WT proinsulin is inhibited, resulting in insulin-deficient diabetes (4). When expressed recombinantly, MIDY proinsulin mutants, including proinsulin- $\mathrm{G}(\mathrm{B} 23) \mathrm{V}$, are not appreciably secreted from heterologous cells (5). Curiously, however, some MIDY mutants expressed in Min6 pancreatic $\beta$ cells, including

Authorship note: Jordan Wright and Xiaofan Wang contributed equally to this work. Conflict of interest: The authors have declared that no conflict of interest exists. Citation for this article: J Clin Invest. 2013;123(7):3124-3134. doi:10.1172/JCI67260. proinsulin- $\mathrm{G}(\mathrm{B} 23) \mathrm{V}$, undergo successful anterograde transport in the secretory pathway, even to the extent of becoming endoproteolytically processed in secretory granules (6). The difference in the secretory fate of proinsulin-G(B23)V in cells lacking a WT proinsulin allele compared with that of $\beta$ cells that express endogenous proinsulin raises the question of whether WT proinsulin could impact transport of the mutant proinsulin- $G(B 23) \mathrm{V}$.

Such a question is also interesting when considering $\mathrm{Tg}$, the major protein product of the thyroid gland that serves as precursor for thyroid hormone synthesis. The large Tg primary structure comprises 3 disulfide-rich upstream regions ("I-II-III") followed by the cholinesterase-like (ChEL) domain (7). Similar to proinsulin, $\mathrm{Tg}$ forms noncovalent homodimers in the ER (8). The ChEL domain functions as an intramolecular chaperone to promote oxidative folding of I-II-III but also functions in Tg homodimerization $(9,10)$. In the disorder known as congenital hypothyroidism with deficient $\mathrm{Tg}$, ChEL is a commonly affected mutation site both in humans (11) and rodent models (12-14). Homozygous $r d w / r d w$ dwarf rats express a single ChEL point mutation [equivalent to G(2298)R of mature mouse $\mathrm{Tg}$ ] and develop thyroid atrophy (15) from thyrocyte cell death (16). When coexpressed, mutant $r d w$ - Tg can cross-dimerize with WT Tg (17).

Understanding the cell biological behaviors of misfolded versions of exportable proteins in the presence of their properly folded partners is of great importance for understanding potential therapeutic approaches to conformational diseases. In this study, we have investigated the selectivity of interactions between 2 ER-retained mutant secretory proteins and their WT counterparts.

\section{Results}

Transdominant retention of a well-folded WT secretory protein partner. To test whether simple ER retention of one homodimerization partner can confer retention to a WT bystander, we expressed various 
A

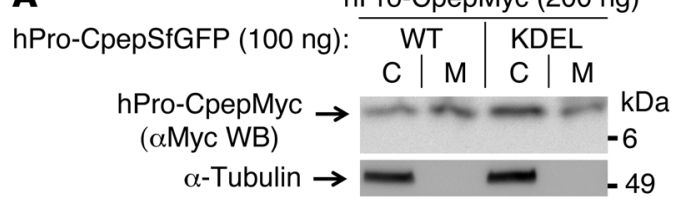

C
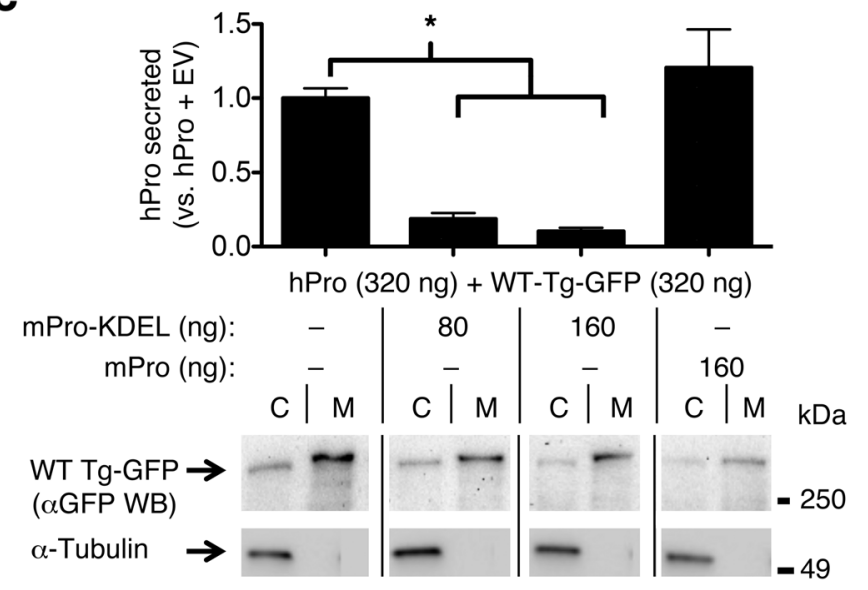

B

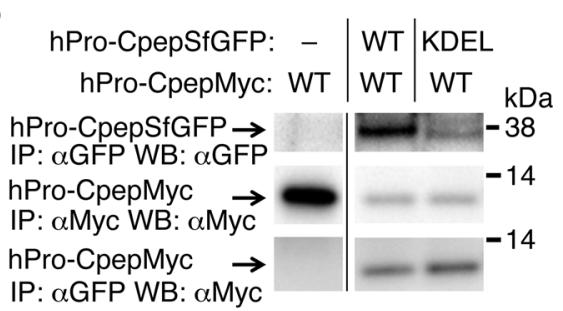

D

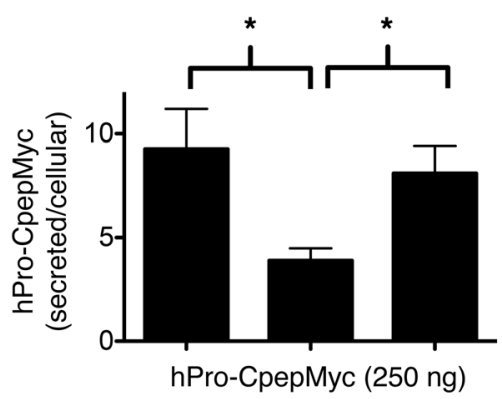

mPro-KDEL (ng):

mPro (ng):

125

500

\section{Figure 1}

Proinsulin-KDEL interacts with and inhibits secretion of WT proinsulin. 293T cells transiently transfected with hPro-CpepMyc were cotransfected with plasmids as indicated. (A) Cell lysates $(C)$ and media (M) were resolved by SDS-PAGE, electrotransfer, and immunoblotting (WB) with antiMyc. The media/cell ratio of hPro-CpepMyc bands was decreased by $58.9 \% \pm 12.8 \%(P=0.003, n=6)$ in cells coexpressing hPro-CpepSfGFPKDEL compared with that in cells coexpressing WT hPro-CpepSfGFP. (B) Cells lysed in TX-CoIP buffer were immunoprecipitated with anti-GFP or anti-Myc and resolved by SDS-PAGE, electrotransfer, and immunoblotting with anti-GFP or anti-Myc as indicated. The top 2 rows demonstrate expression of the indicated proteins, and the bottom row demonstrates ColP. Gels are representative of 3 independent experiments. (C) Cells transiently expressing WT hPro plus TgGFP were cotransfected with plasmids as indicated. The media collected overnight were analyzed by hPro-specific RIA. TgGFP in the same cell lysates and media was analyzed by SDS-PAGE, electrotransfer, and immunoblotting with anti-GFP. The media/cell ratio of TgGFP bands in cells coexpressing mPro-KDEL exhibited no significant change compared with that from cells coexpressing WT mPro $(1.9 \pm 0.2$ vs. $2.1 \pm 0.8 ; P=0.3, n=5)$. In $\mathbf{B}$ and $\mathbf{C}$, noncontiguous lanes from the same gel are shown. (D) Cells transiently expressing hPro-CpepMyc were cotransfected with plasmids expressing mPro-KDEL or mPro. Media were collected overnight, and cell lysates were analyzed by hPro-specific RIA. The data in $\mathbf{C}$ and $\mathbf{D}$ represent mean $\pm \mathrm{SEM}$, each from $\geq 4$ independent transfections. ${ }^{*} P<0.05$.

epitope-tagged proinsulin constructs: human proinsulin (hPro) bearing or not bearing a Myc- or SuperfolderGFP-tag within the C-peptide sequence (hPro-CpepMyc and hPro-CpepSfGFP, respectively) or mouse proinsulin (mPro). Epitope tagging the C-peptide does not significantly affect the folding or ER export of WT proinsulin (18). We also expressed proinsulin(s) bearing a C-terminal KDEL sequence reported to confer proinsulin retention within the ER (19). All of these recombinant proinsulins were comparably expressed, and the expression level for each protein could be experimentally controlled (although the mutant hProG[B23] $\mathrm{V}$-CpepMyc is less stable; see below), as it was proportional to the amount of plasmid DNA included in our transfections (Supplemental Figure 1A; supplemental material available online with this article; doi:10.1172/JCI67260DS1). hPro-CpepSfGFP-KDEL was retained intracellularly in INS1 $\beta$ cells (Supplemental Figure 1B), primarily in the same compartment as that marked by a ER-RFP, a red fluorescent protein bearing the KDEL retention signal (6).

Interestingly, when cotransfected with hPro-CpepSfGFP-KDEL plasmid, the intracellular content of WT hPro-CpepMyc increased, and its secretion decreased (Figure 1A). Intracellular WT hProCpepMyc coimmunoprecipitated with hPro-CpepSfGFP-KDEL, perhaps to an even greater extent than its coprecipitation with WT hPro-CpepSfGFP (Figure 1B, bottom row). Nevertheless, even as increasing doses of mPro-KDEL dramatically inhibited secretion of WT hPro (Figure 1C, top, measured by hPro radioimmunoassay [RIA]), secretion of WT TgGFP was largely unaffected in the same cells (Figure 1C, bottom). These results suggest that blockade of export of the well-folded WT secretory protein partner involves specific interactions with its ER-retained dimerization partner. Indeed, as shown in Figure 1D, secretion of WT hPro-CpepMyc was largely restored upon overexpression of WT mPro "competitor," even in the presence of mPro-KDEL.

Misfolded mutant secretory protein also assembles with and impairs secretion of its WT partner. We have previously suggested that, in the autosomal dominant disease called MIDY, misfolded mutant proinsulin can inhibit WT proinsulin export by recruitment of the WT gene product into aberrant protein complexes within the $\operatorname{ER}(5,18)$. Indeed, when WT hPro-CpepMyc was coexpressed with misfolded mutant $\mathrm{mProG}(\mathrm{B} 23) \mathrm{V}$ in place of WT mPro, the WT hPro-CpepMyc secretion was impaired in trans (Figure 2A). By contrast, the mutant $r d w$ Tg functions as an autosomal recessive allele (15); nevertheless, surprisingly, we found that coexpression of $r d w$-Tg also could 
A
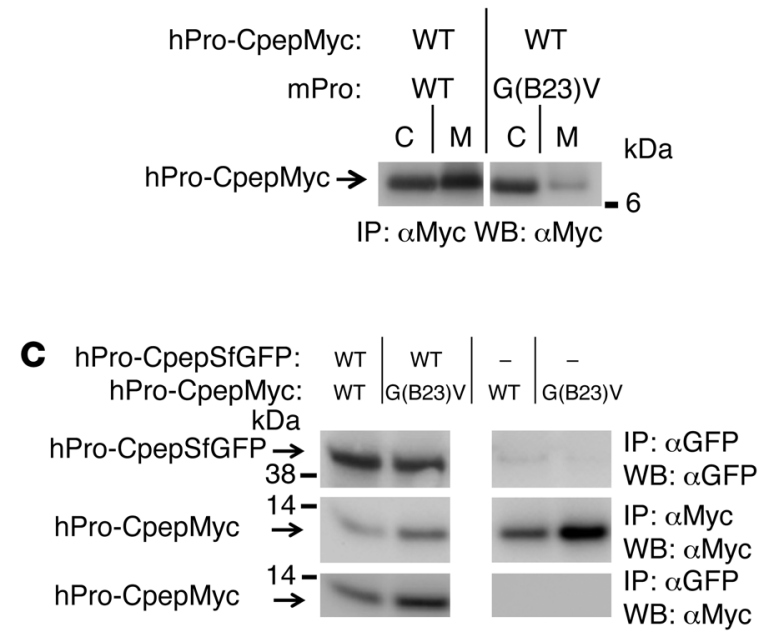

B

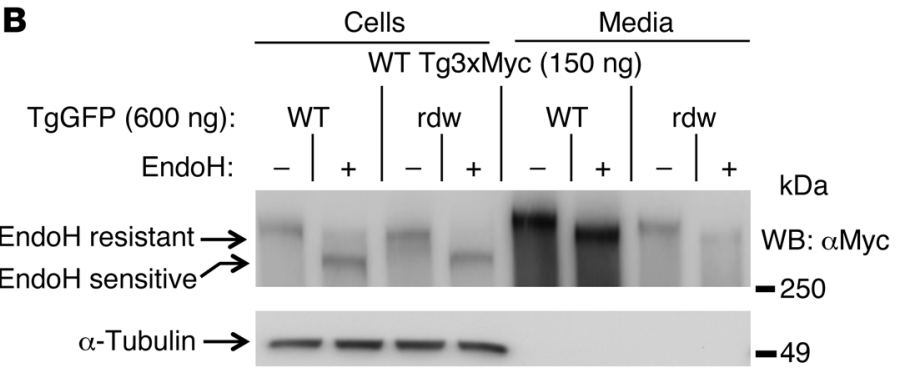

D

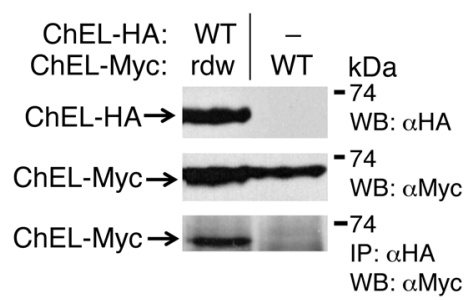

Figure 2

Cross-dimerization of mutant/WT proinsulin and mutant/WT Tg. 293T cells were transiently cotransfected with plasmids expressing the indicated proinsulin or Tg variants. (A) At 48 hours after transfection, cell lysates and overnight media were collected; both were immunoprecipitated with anti-Myc (to prepurify the antigen) and then analyzed by SDS-PAGE, electrotransfer, and immunoblotting with anti-Myc. The media/cell ratio of hPro-CpepMyc bands from cells coexpressing mPro-G(B23)V decreased $66.1 \% \pm 1.9 \%(P<0.001, n=4)$ compared with that of WT mPro. (B) At 48 hours after transfection, cell lysates and overnight media were collected, treated with or without EndoH, and were analyzed by immunoblotting with anti-Myc. The media/cell ratio of WT Tg3xMyc bands in cells coexpressing $r d w$-TgGFP decreased $74.1 \% \pm 3.3 \%(P<0.001, n=4)$ compared with that of WT TgGFP. (C) At 48 hours after transfection, cells lysed in TX-ColP buffer were immunoprecipitated with anti-GFP or antiMyc and were analyzed by Western blotting with anti-GFP or anti-Myc, as indicated. (D) Cells transiently coexpressing WT secretory ChEL-HA plus secretory mutant rdw-ChEL-Myc or secretory WT ChEL-Myc (9) were cultured in the presence of brefeldin A ( 5 hours, $5 \mu \mathrm{g} / \mathrm{ml})$ to allow intracellular coincubation of the coexpressed constructs. Cells were then lysed in NP40-ColP buffer and analyzed by immunoblotting with or without immunoprecipitation, as indicated. For $\mathbf{C}$ and $\mathbf{D}$, the top 2 rows demonstrate expression of protein partners, and the bottom row demonstrates ColP. Gels in $\mathbf{C}$ and $\mathbf{D}$ are representative of $n \geq 3$ experiments. In $\mathbf{A}$ and $\mathbf{C}$, noncontiguous lanes from the same gel are shown.

greatly decrease the secretion of WT Tg3xMyc (Figure 2B), with the extent of this dominant-negative inhibition dependent upon the relative abundance of mutant versus WT gene products (see below). Unlike secreted WT Tg3xMyc, WT Tg3xMyc retained intracellularly in the presence of coexpressed $r d w$-Tg was recovered in an endoglycosidase $\mathrm{H}$-sensitive (EndoH-sensitive) state, indicating molecules that had not advanced to the Golgi complex (Figure 2B). The $r d w$-Tg has a point mutation in the ChEL domain that is involved in $\mathrm{Tg}$ homodimerization $(10,17)$. To test whether the rdw ChEL domain can indeed associate with the WT Tg ChEL domain, WT ChEL-HA was coexpressed with rdw-ChEL-Myc. Similar to the situation with the mutant proinsulin hProG(B23)V-CpepMyc (Figure 2C, bottom), we found that mutant rdw-ChEL could coprecipitate with its WT dimerization partner (Figure 2D, bottom).

WT mutant cross-dimerization offers secretory rescue to the mutant gene product. In INS1 cells, WT hPro-CpepSfGFP efficiently reached secretory granules and was well secreted, whereas hProC(A7)YCpepSfGFP (which harbors the same mutation as that found in the Akita mouse) was retained in the ER (Figure 3A) rather than being secreted (Figure 3A) (20). Interestingly, however, recombinant hProG(B23)V-CpepSfGFP showed intermediate behavior, with partial ER retention and a partial secretory granule distribution, and an intermediate level of secretion - consistent with a previous report (6). This stands in contrast to findings that secretion of mutant hProG(B23)V is negligible in heterologous cells that do not express endogenous WT proinsulin (5). We therefore considered whether secretion of hProG(B23)V might be improved in cells expressing a WT proinsulin partner. To test this, we expressed WT hPro-CpepSf-
GFP in the mouse pituitary cell line AtT20 (which forms secretory granules containing ACTH but does not express proinsulin). In this cell line, WT hPro-CpepSfGFP colocalized with endogenous ACTH in secretory granules that accumulate at the distal tips of cellular processes (Figure 3B). When expressed by itself, hProG(B23)VCpepSfGFP exhibited primarily an ER distribution and did not reach secretory granules (Figure $3 \mathrm{~B}$ ). However, when coexpressed with WT hPro-CpepMyc, the hProG(B23)V-CpepSfGFP was partially rescued, becoming visible in secretory granules (Figure $3 \mathrm{~B}$ ). These results indicate that expression of WT proinsulin enhances intracellular transport of mutant hProG(B23)V.

To determine the selectivity of this rescue, $293 \mathrm{~T}$ cells were cotransfected with fixed equimolar amounts of hProG(B23)VCpepMyc and $r d w$-TgGFP and simultaneously cotransfected with either WT mPro or WT Tg (empty vector was included to keep constant the total DNA in each transfection). Secretory rescue of hProG(B23)V was provided selectively by WT mPro (Figure 4A, top; confirmed by immunoblotting in Figure 4B) but not by WT $\operatorname{Tg}$ (Figure 4A, top), as measured by hPro-specific RIA. Conversely, rescue of $r d w$-TgGFP secretion was conferred upon coexpression of WT Tg (17) but not by WT mPro (lane M, Figure 4A, bottom). This phenotype involved authentic intracellular transport through the secretory pathway (rather than cell death), as rescue was blocked in cells treated with brefeldin A (which blocks anterograde transport; Figure 4C). Thus, not only can an ER-retained dimerization partner impair secretion of its WT counterpart (Figures 1 and 2), but expression of a WT dimerization partner augments secretion of its misfolded counterpart (Figures 3 and 4). 
WT

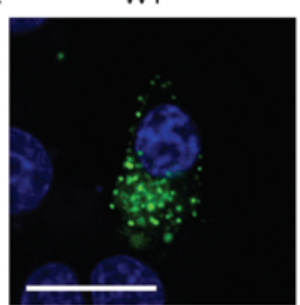

G(B23)V
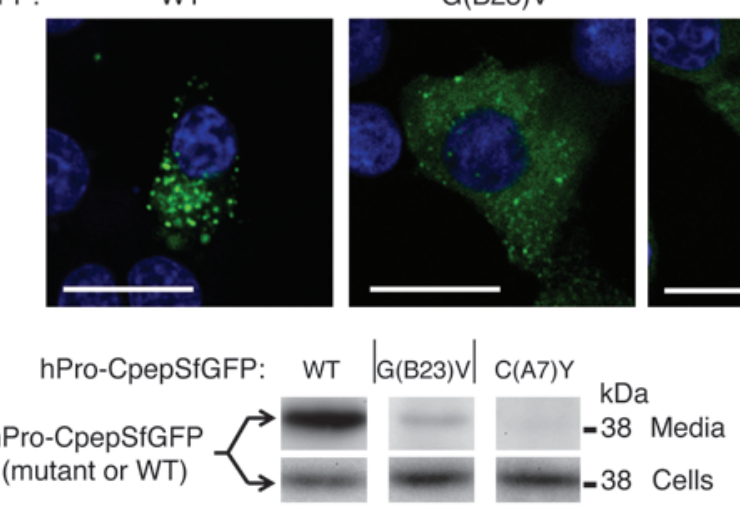

IP: $\alpha$ GFP WB: $\alpha$ GFP

B

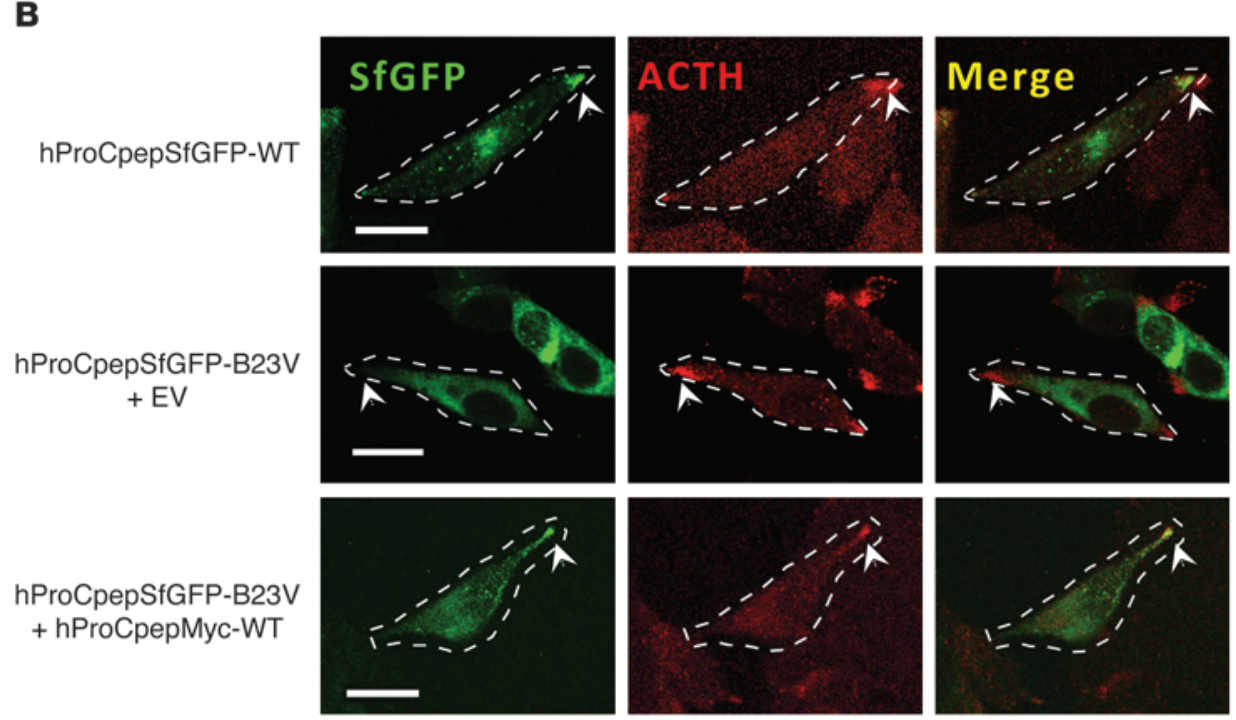

$\mathrm{C}(\mathrm{A} 7) \mathrm{Y}$

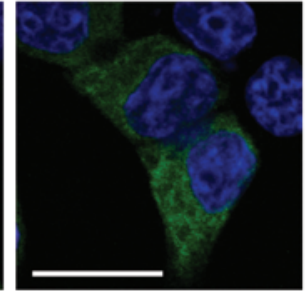

Cells

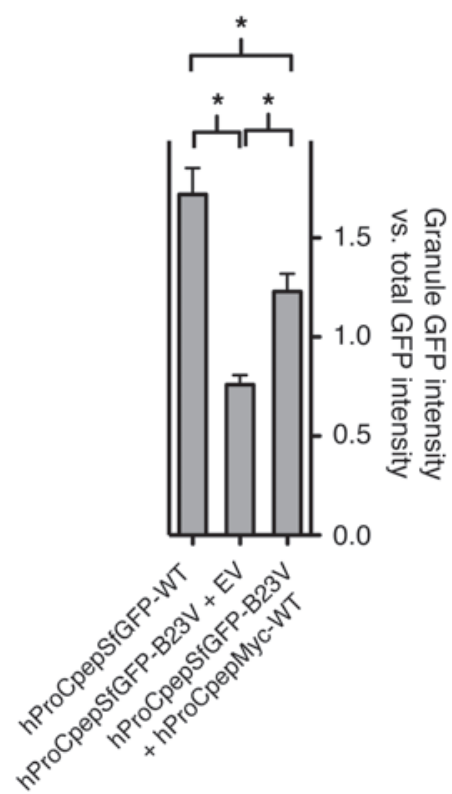

Figure 3

Intracellular distribution of mutant proinsulins in regulated secretory cells coexpressing or not coexpressing WT proinsulin. (A) Cultured INS1 pancreatic $\beta$ cells (that express endogenous proinsulin) were transiently transfected to express WT or mutant hPro-CpepSfGFP, as indicated. Fixed cells (counterstained with DAPI) were examined by confocal microscopy for the distribution of SfGFP-containing peptides (scale bar: $20 \mu \mathrm{m}$ ). The cell lysates and overnight bathing media were collected, immunoprecipitated with anti-GFP, and analyzed by immunoblotting with anti-GFP to examine secretion efficiency. Noncontiguous lanes from the same gel are shown. The media/cell ratio for WT, G(B23)V, and C(A7)Y hPro-CpepSfGFP bands was $14.8 \pm 3.8,0.74 \pm 0.04$, and $0.16 \pm 0.06$, respectively $(P<0.05$ for all groups, $n=4)$. (B) Cultured AtT20 pituitary corticotroph cells (that do not express endogenous proinsulin) were transiently cotransfected with one of three different plasmid combinations, as indicated. Fixed cells were examined by confocal fluorescence for the distribution of SfGFP-containing peptides (green) and immunofluorescence to localize ACTH-containing secretory granules (red) at the tips of cell (arrowheads; scale bar: $20 \mu \mathrm{m}$ ). Cell boundaries were defined from phase-contrast images (data not shown). Enrichment of average GFP intensity in the secretory granule region was compared with average GFP intensity in nongranule regions. Data represent mean \pm SEM from 30 to 38 separately imaged cells for each of the 3 respective transfection conditions. ${ }^{*} P<0.05$.

WT dimerization partners dose-dependently stabilize their misfolded counterparts for secretory rescue. To examine the stability of hProG(B23)V-CpepMyc, we used metabolic pulse labeling of cells with ${ }^{35} \mathrm{~S}$-amino acids and measured the fraction of newly synthesized protein remaining at 20 hours after synthesis. hProG(B23)VCpepMyc stability was significantly increased by coexpression of WT mPro (Figure 5A). In parallel, WT Tg3xMyc increased both the intracellular as well as secreted amounts of $r d w$-TgGFP - indeed, these observations were dependent on the dose of WT Tg3xMyc (Figure 5B). Rescue of hProG(B23)V-CpepMyc also was observed with increasing concentrations of coexpressed WT mPro (Figure 5C).
Notably, rescue appeared more dependent upon the ratio between WT and mutant proteins than the absolute amount of WT protein expressed, because increasing rescue was also observed with decreasing expression of misfolded mutant $r d w$-TgGFP rather than when the amount of WT Tg3xMyc was raised (Figure 5D).

Rescue of mutant and blockade of WT proinsulin and Tg occur within the (patho)physiological context. To determine whether the effects of altering WT/mutant stoichiometric ratio in heterologous cells also are observed in pancreatic $\beta$ cells, we expressed 2 different doses of hProG(B23)V-CpepSfGFP in INS1E cells that are known to secrete endogenous proinsulin and insulin under basal conditions and 
A

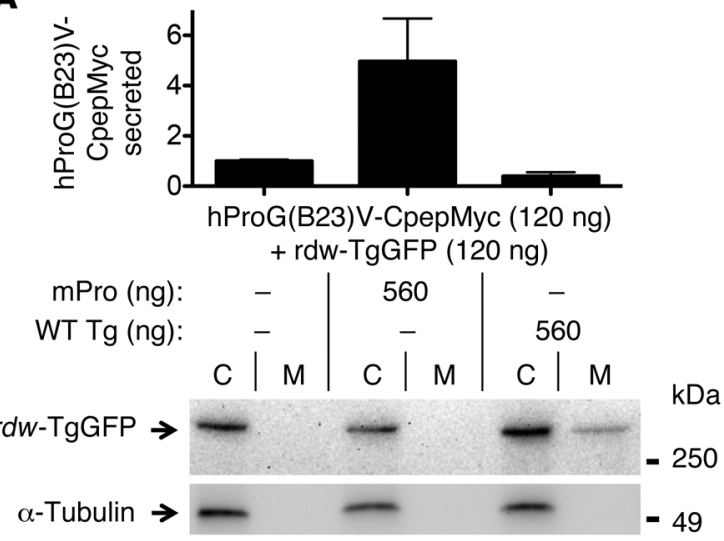

B

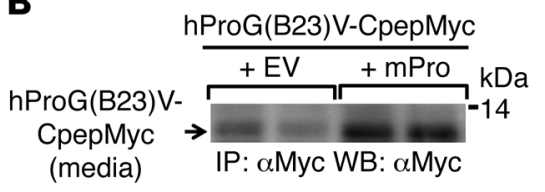

C

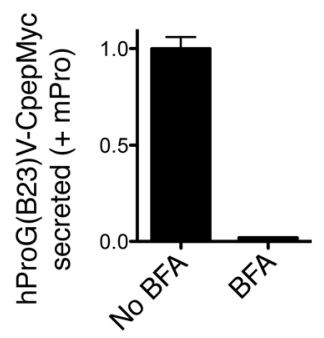

Figure 4

Cross-dimerization as a basis for secretory rescue of mutant proinsulin or Tg is specific to respective WT partners. (A) 293T cells transiently expressing both mutant proinsulin and mutant Tg were cotransfected with either WT mPro or WT Tg. The media were collected overnight, and cells were lysed; proinsulin secretion was quantified by hPro-specific RIA. Data represent mean \pm SEM relative to cells lacking mPro or WT $\operatorname{Tg}(P=0.07, n=3)$. From the same cell lysates and media, secretion of $r d w$-TgGFP was analyzed by SDS-PAGE, electrotransfer, and immunoblotting with anti-GFP. (B) At 48 hours after cotransfection, overnight secretion of mutant $\mathrm{hProG}$ (B23) $\mathrm{V}$-CpepMyc (in duplicate) was measured by immunoprecipitation and immunoblotting with anti-Myc. The results shown in $\mathbf{A}$ and $\mathbf{B}$ are representative of 3 separate experiments. EV, empty vector. (C) At 48 hours after transfection, cells cotransfected as indicated were either untreated or treated with $5 \mu \mathrm{g} / \mathrm{ml}$ brefeldin A (BFA). After 5 hours, the media were collected and analyzed by hPro-specific RIA. The data shown are mean values \pm range from 2 independent measurements.

at greater levels in response to elevated glucose. Unsurprisingly, INS1E cells transfected with less plasmid expressed less mutant proinsulin (Figure 5E). Interestingly, INS1E cells expressing less mutant proinsulin (and thus a higher WT/mutant stoichiometric ratio) exhibited higher fractional secretion of the mutant protein under both basal and glucose-stimulated conditions, including both unprocessed hProG(B23)V-CpepSfGFP and its CpepGFPprocessing product (Figure $5 \mathrm{E}$ ).

We next wished to extend these findings to tissues of animals with diseases of protein misfolding in the ER. First, we looked directly at the rescue of mutant $r d w-\operatorname{Tg} 3 \times \mathrm{XMyc}$ in thyroid tissue from animals lacking or bearing WT Tg. For this, we prepared an $r d w$ - $\mathrm{Tg} 3 \mathrm{xMyc}$ transgene whose expression in the thyroid gland was driven by a $\mathrm{Tg}$ promoter. The $r d w$ - $\mathrm{Tg}$ protein was expressed in thyroid tissue of otherwise WT C57BL/6 mice or mutant $\operatorname{cog} / \operatorname{cog}$ mice (with homozygous expression of mutant Tg-L2263P) in a C57BL/6 background. Both strains of mice expressed the $r d w$-Tg protein in the thyroid gland (Supplemental Figure 2), and both strains can iodinate secreted proteins (21). However, in thyroid tissue from the $\operatorname{cog} / \operatorname{cog}$ background (lacking WT Tg), no $r d w$-Tg could become iodinated, indicating an inability of $r d w$-Tg to reach the iodination site (Figure 6A). By contrast, in thyroid tissue of C57BL/6 control mice expressing WT Tg, some mutant $r d w$-Tg was rescued based on the ability to become iodinated - moreover, the iodinated $r d w-\mathrm{Tg}$ was endoH resistant, indicative of molecules that had undergone normal intracellular transport through Golgi/post-Golgi compartments (Figure 6A).

We also wished to determine whether decreased WT/mutant stoichiometry can promote blockade of the WT gene product. For this, we examined mice expressing the Akita-like mutant hProC(A7)Y-CpepGFP transgene (22), with deletion of either one allele or homozygous knockout of endogenous Ins2 to protent is heterogeneous in the population of islet $\beta$ cells $(24,25)$. Nevertheless, in Ins2 $2^{-/}$mice, as the ratio of WT/mutant proinsulin decreased, the fraction of cells exhibiting endogenous WT proinsulin in an ER-staining pattern increased - and this effect was apparent in mice matched for random blood glucose levels $\leq 250 \mathrm{mg} / \mathrm{dl}$ (Figure 6B). These findings demonstrate that, in tissues from actual conformational disease models, both mutant secretory protein rescue (Figure 6A) and WT secretory protein blockade (Figure 6B) are influenced by the ratio of WT/mutant secretory protein in the ER.

Can secretory rescue and secretory blockade occur simultaneously? To examine whether both rescue (of mutant) and blockade (of WT) dimerization partners can occur simultaneously, we first transfected 293T cells with WT mPro or hProG(B23)V or both. We then independently measured mPro and hPro secretion by species-specific immunoassay. Secretion of WT mPro was significantly diminished by the presence of hProG(B23)V (Figure 7A). Remarkably, from the same cells, hProG(B23)V secretion was improved by the coexpression of WT mPro (Figure 7A). Similarly, when transfecting with progressively increasing ratios of WT Tg3xMyc to mutant $r d w$-TgGFP, $r d w$-TgGFP secretion improved (Figure 7B), whereas WT Tg3xMyc was markedly diminished when the ratio favored $r d w$-TgGFP (Figure 7B). Intermediate ratios showed both rescue and blockade (Figure 7B). Both sets of data in Figure 7 indicate that these effects occur simultaneously in the same cells.

Is secretory rescue of misfolded proinsulin unique to the $G(B 23) V$ substitution? Multiple MIDY mutants cause dominant-negative blockade of WT proinsulin export (5). Structurally, MIDY can be subdivided into those mutants that cause the gain or loss of a Cys residue to create an unpaired cysteine, and other mutants affecting conserved hydrophobic residues that perturb disulfide pairing of the 


\section{A}
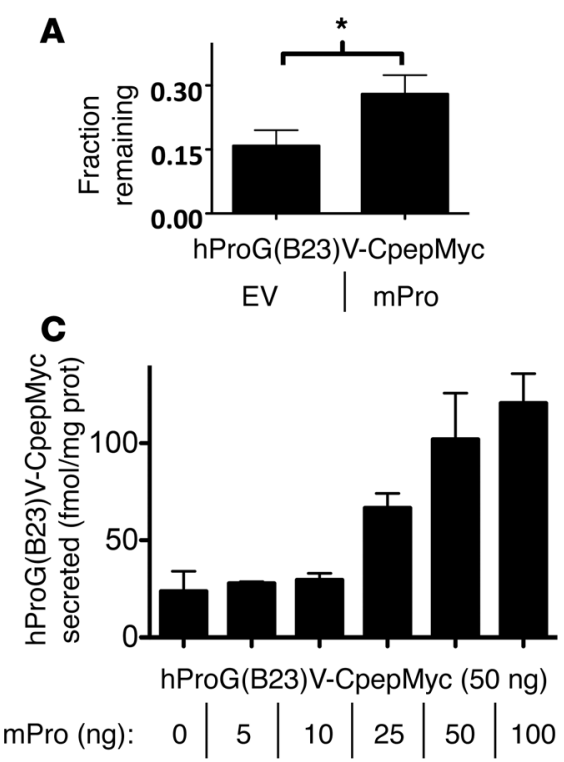

B

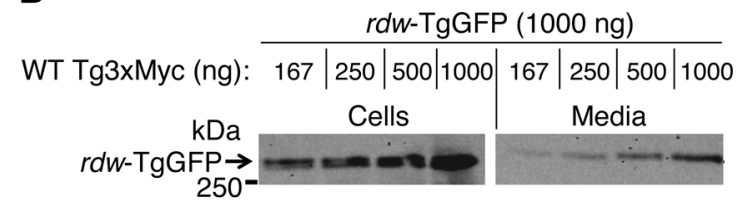

D

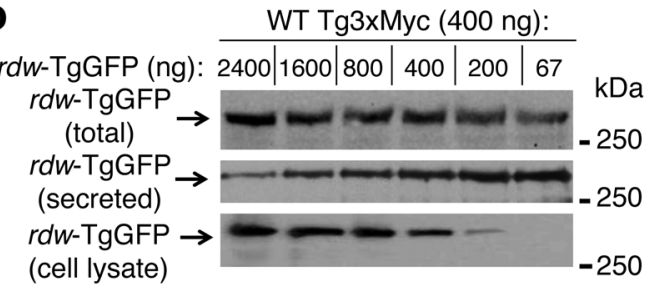

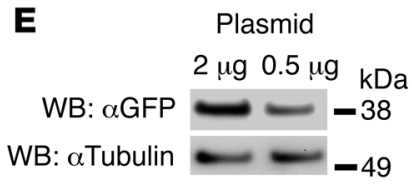

hProG(B23)V-CpepSfGFP:

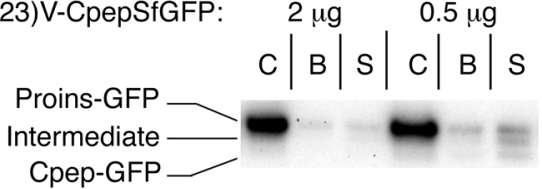

WB: $\alpha$ GFP

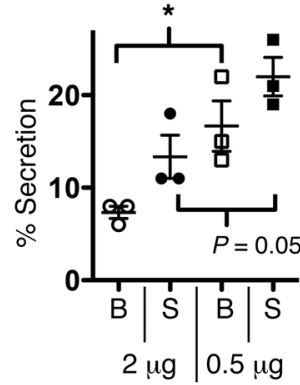

Plasmid/well

\section{Figure 5}

Secretory rescue and stabilization of mutant proinsulin or Tg by their WT counterparts is linked to the WT/mutant expression ratio. (A-D) 293T cells were transiently cotransfected with the indicated plasmid combinations, with empty vector added to keep total DNA per well constant. (A) Total mutant proinsulin recovery at 20 hours after synthesis was measured by pulse chase (see Methods). Protein stability was quantified by band recovery at 20 hours chase relative to that at time 0 (mean $\pm \mathrm{SEM}, n=4,{ }^{\star} P<0.05$ ). (B) Overnight media and cell lysates were analyzed by immunoblotting with anti-GFP (normalized to total cellular protein). Representative blots from 3 experiments are shown. (C) Overnight media were collected, and hPro secretion (normalized to total cellular protein) was measured by RIA. Data represent mean \pm range from 2 independent experiments. (D) Overnight media and cell lysates (bottom 2 rows) or combined lysate and media (top row) were analyzed by immunoblotting with antiGFP (normalized to total cellular protein). Representative blots from 3 experiments are shown. (E) INS1E cells were transfected with 2 or $0.5 \mu \mathrm{g}$ plasmid expressing mutant proinsulin; the cellular levels of hProG(B23)V-CpepSfGFP are shown at left. Cell lysates and basal secretion (B) and glucose-stimulated secretion (S), normalized for hProG(B23)V-CpepSfGFP protein expression (1\% of total for $2 \mu \mathrm{g}$ transfection; $3 \%$ of total for $0.5 \mu \mathrm{g}$ transfection), were analyzed by immunoblotting with anti-GFP. Percentage secretion was quantified as total GFP signal in media over total in cells. The data represent mean \pm SEM, from 3 independent experiments. ${ }^{*} P<0.05$.

natural cysteine partners (4). To see which class of mutants could be rescued by coexpression of WT proinsulin, we compared secretion of a series of coexpressed MIDY mutants. Notably, secretion of most proinsulin mutants containing an unpaired cysteine, such as $\mathrm{C}(\mathrm{A} 6) \mathrm{Y}, \mathrm{C}(\mathrm{B} 19) \mathrm{H}$, or the $\mathrm{C}(\mathrm{A} 7) \mathrm{Y}$ mutant responsible for diabetes in the Akita mouse, was not improved by coexpression of WT proinsulin (Figure 8). By contrast, secretion of several other misfolded MIDY proinsulins [e.g., A(SP24)D, H(B5)D, G(B8)S] was rescued in addition to $\mathrm{G}(\mathrm{B} 23) \mathrm{V}$. These data suggest that this mechanism does not work in all cases; nevertheless, cross-dimerization of proinsulin (Figure 1B and Figure 2C) or Tg (Figure 2D) exhibits plasticity, highlighting the potential for intracellular transport rescue of a variety of misfolded exportable proteins by their WT counterparts.

\section{Discussion}

There are a large number of disorders linked to misfolding, ER entrapment (26), and degradation of exportable proteins (27), and several new approaches have been proposed for the development of therapies for these diseases. Some of these proposals include pharmacological modification of the rate of protein synthesis to avoid overloading protein folding capacity (28), others involve manipulation of the intraluminal ER ionic milieu $(29,30)$ or modulating ER-associated degradation (ERAD) (31), and still others involve pharmacologic chaperones (32) or modulators of endogenous ER chaperone activity $(33,34)$, including preemptive induction of unfolded protein response (35). Each of these therapies is designed to manipulate the ER quality control environment, altering the ratio of protein folding to protein folding capacity. 
A
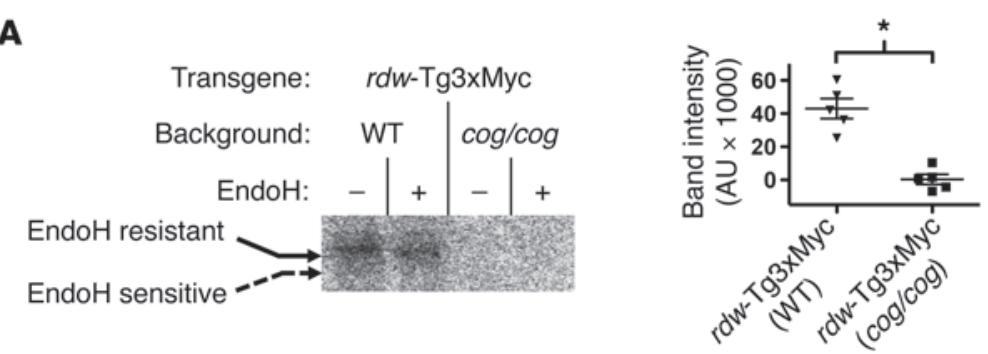

B
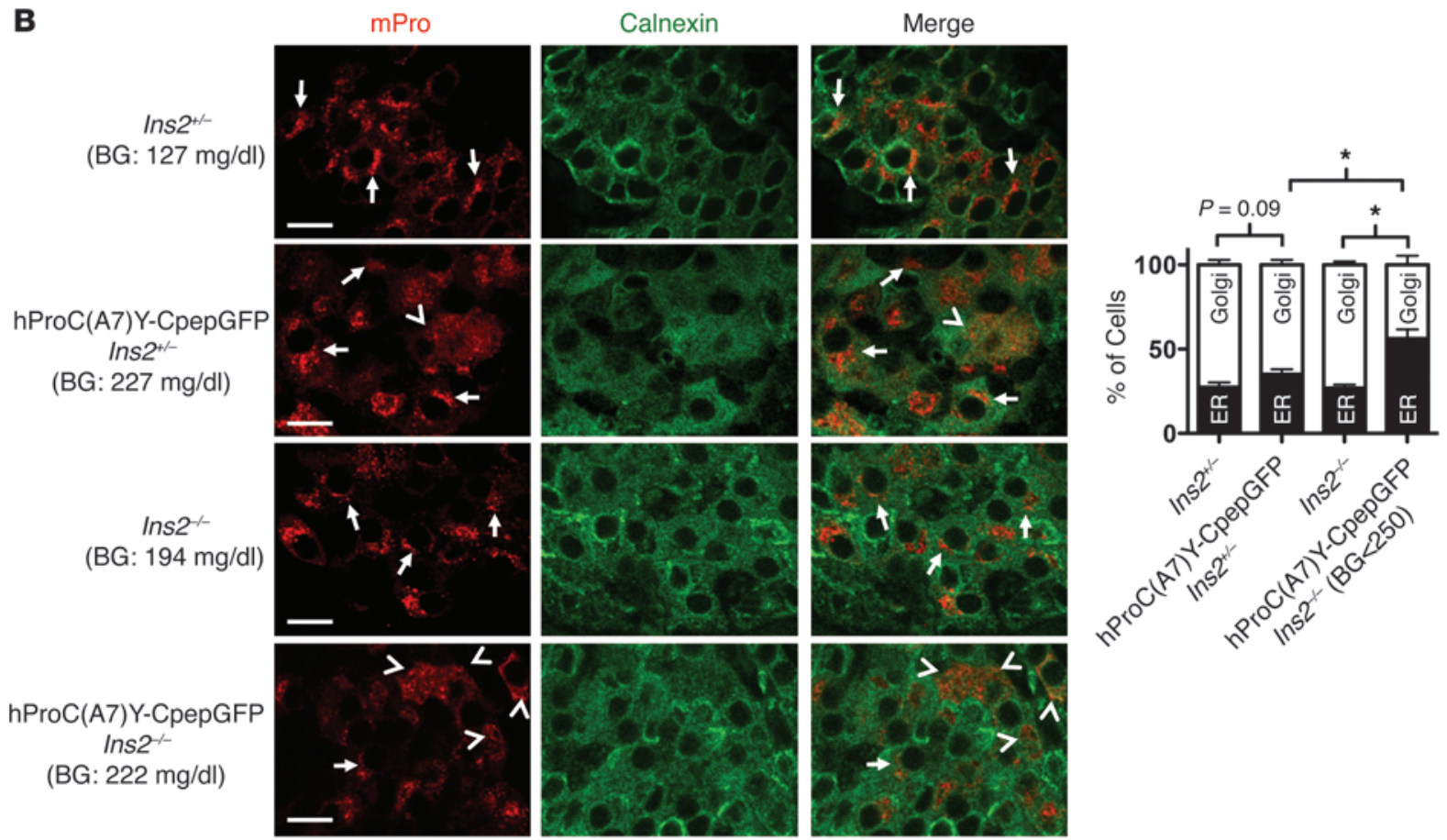

\section{Figure 6}

Rescue of mutant Tg and blockade of WT proinsulin in primary tissue from animal models of disease. (A) Lobules of thyroid glands were freshly prepared from mice of the indicated genotypes. Secretory proteins delivered for posttranslational iodination were labeled by incubation of thyroid lobules with $1.0 \mu \mathrm{Ci} / \mu \mathrm{l} \mathrm{Na}{ }^{125}$ for 30 minutes, as described in Methods. The thyroid lobules were then lysed and immunoprecipitated with anti-Myc. The immunoprecipitates were either mock-digested or digested with EndoH, as in Figure 2B, and then analyzed by SDS-PAGE and autoradiography. ${ }^{*} P<0.05$. (B) Pancreata from 6-week-old mice, with the genotypes indicated, were fixed in paraffin, sectioned, deparaffinized, and immunostained with antibodies specific to mPro (red) and calnexin to mark the ER (green). From confocal microscope images (scale bar: $10 \mu \mathrm{m}$ ), a blinded reader scored the localization of WT mPro in each $\beta$ cell as either a predominant juxtanuclear crescent of increased intensity (Golgi, consistent with previous reports, refs. 20, 23; e.g., see arrows) or mainly colocalized with calnexin (ER; e.g., see arrowheads). Quantitation of these data is shown as mean \pm SEM from $n=5$ mice with 5 islets per mouse. BG, blood glucose. ${ }^{*} P<0.05$.

In addition to these critical features, we also note that there has been unrecognized selection pressure for exportable proteins to evolve as oligomeric species (36). Indeed, even upon initial description of the ER hsp70 chaperone, BiP, its ability to confer ER retention was found to be linked to its selective association with unassembled subunits of exportable protein oligomers (37). Oligomerization limits chaperone rebinding and helps to relieve ER entrapment $(38,39)$, often by limiting exposure of unpaired Cys residues that can frequently be associated with ER retention (40-42). Typically, monomer folding precedes oligomerization (43), but there are examples in which oligomer formation may be a very early folding step (44). Either way, for many exportable proteins, achieving an oligomeric state is a critical decision point in determining anterograde transport versus ERAD (45).
It has been shown that expression of misfolded mutant proteins has the potential to cause WT (bystander) dimerization partners to be retained in the ER $(5,18,46-49)$. Protein-specific rather than general enhancement - lowering the levels of the mutant partner (50) and raising WT levels - has been proposed as one of the more efficacious therapeutic approaches, by maintaining general protein homeostasis while allowing escape from ER entrapment of a specific disease-linked gene product $(51,52)$. In this study, we emphasize that one potential consequence of raising the WT/ mutant protein ratio is that an increased fraction of mutant protein may cross-dimerize with WT, allowing for novel, protein-specific enhancement of protein export. Our findings are unequivocal, because we have selectively epitope tagged the respective WT and mutant partners and experimentally controlled the expression levels of the respective products (Figures 1 and 5-7). In this 
A
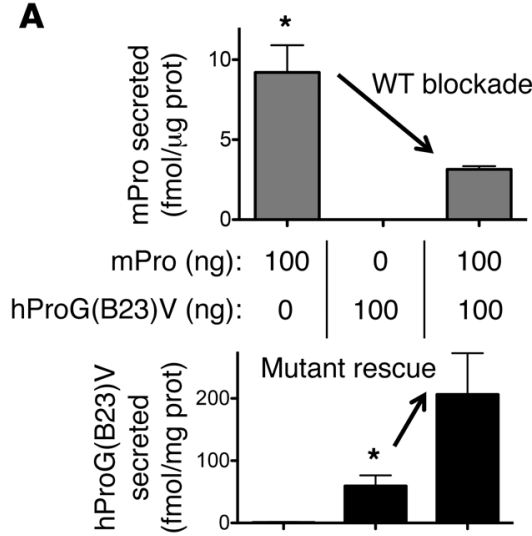

B

rdw-TgGFP (ng): $\frac{\text { WT Tg3xMyc (400 ng) }}{1600|800| 400 \mid 200}$

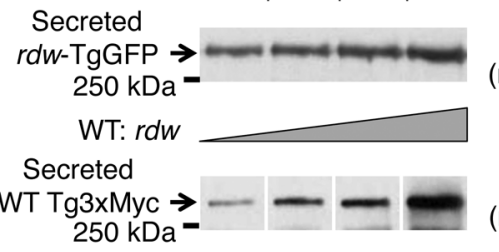

WB: $\alpha$ GFP

(measuring rescue)

WB: $\alpha$ Myc

(measuring blockade)

\section{Figure 7}

Bidirectional consequences of interactions between mutant and WT cross-dimerization partners. (A) The overnight bathing media from cells cotransfected with mutant hPro-G(B23)V and WT mPro were selectively probed for simultaneous secretion of mPro (by ELISA) and hPro (by $\mathrm{RIA}$ ); both assays (normalized to total cell protein) are entirely species specific. The data represent mean \pm SEM of a minimum of 3 independent transfections; the arrows in the top and bottom panels demonstrate blockade and rescue, respectively. ${ }^{*} P<0.05$. (B) The overnight bathing media from cells cotransfected with $r d w$-TgGFP and WT Tg3xMyc were resolved by SDS-PAGE and selectively probed for simultaneous secretion of mutant Tg (by specific immunoblotting with anti-GFP) and WT Tg (by specific immunoblotting with anti-Myc), normalized to total cellular protein. For the bottom panel, noncontiguous lanes from the same gel are shown. Note that, in cotransfection, $r d w$ - Tg secretion becomes enhanced while WT Tg secretion becomes inhibited, whereas, in single transfections, $r d w$-Tg is not secreted and WT Tg is well secreted. The data shown are representative of 3 independent experiments.

report, we have studied proinsulin mutants causing MIDY and $\mathrm{Tg}$ mutants causing congenital hypothyroidism as 2 genetically unrelated representatives of a broad class of conformational diseases of exportable proteins. The organs affected by these diseases appear quite different in terms of their ability to expand tissue mass, and they might have different intrinsic susceptibility to ER stress (a goiter can grow large, whereas expansion of pancreatic $\beta$ cell mass is more limited), yet studying these molecules in parallel has allowed us to address persistent questions about the extent to which phenotypes linked to cross-dimerization are protein specific and the extent to which the pathogenesis of these diseases is secondary to generalized ER stress.

In this study, we demonstrate that retention of proinsulinKDEL, via a mechanism involving neither misfolding nor ER stress, is sufficient to induce export blockade of its WT dimerization partner (Figure 1, A and C) in conjunction with direct physical association between the partners (Figure 1B). Also, further addition of WT proinsulin competes with the proinsulin-KDEL to allow escape from this blockade (Figure 1D). In the same cells, WT Tg secretion continues unimpeded in the face of proinsulin blockade, demonstrating specificity (Figure 1C). We have every reason to believe that the initial pathogenesis of MIDY is based on a similar underlying cell biological principle: in the case of mutant versions of exportable proteins, chaperone-mediated retention of the misfolded gene product is the rule (53). While formation of misfolded protein complexes was once thought to be nonspecific (54), we have found that expression of the proinsulin-G(B23)V mutant selectively coprecipitates its WT partner (Figure 2C) and blocks secretion of that partner (Figure 2A) while neither associating with nor blocking WT Tg in the same cells (Figure 1C and data not shown). This dominant effect on WT proinsulin appears to account for the dominant inheritance of MIDY (Figure 9), producing insulin deficiency from the WT allele (4). Moreover, expressing the Akita-like hProC(A7)Y-CpepGFP transgene in mice first with heterozygous and then with homozygous loss of endogenous Ins2 (i.e., changing the relative expression of the 2 protein partners) results in progressive blockade of remaining WT proinsulin, independent of changes in random blood glucose (Figure 6B).

Despite an autosomal recessive pattern of inheritance, we found surprisingly similar molecular behavior for the genetically unrelated $\mathrm{Tg}$ protein. The $r d w$-Tg, which bears a mutation in the ChEL domain and causes thyrocyte cell death only when expressed in homozygotes (16), also exhibits cross-dimerization with WT Tg in heterozygotes (17). Herein, we show that the molecular mechanism involves direct interactions between the mutant and WT dimerization (ChEL) domains (Figure 2D). Remarkably, we found that expression of the recessive $r d w$-Tg can also dominantly block export of its WT Tg partner (Figure 2B), and this phenotype is linked to lowering the $\mathrm{WT} /$ mutant protein ratio (Figure $7 \mathrm{~B}$ ). The fact that heterozygosity does not generate hypothyroidism in vivo indicates that $r d w /+$ rats express a $\mathrm{Tg}$ protein ratio favoring the WT gene product. Further, when expression of the WT gene product is favored, rescue phenotypes become apparent. Such rescue was directly demonstrated in thyroid tissues of mice expressing a mutant $r d w$-Tg3xMyc transgene in a WT genetic background: unlike in $\operatorname{cog} / \operatorname{cog}$ mice, $r d w$-Tg3xMyc acquired endoH resistance and even became iodinated in animals making endogenous WT Tg (Figure 6A). Moreover, rescue is also observed for proinsulin mutations that are ordinarily transmitted with autosomal dominant inheritance. In pancreatic $\beta$ cells, partial rescue of proinsulin-G(B23)V to secretory granules was already in evidence (Figure 3A), conferring enhanced secretion under both basal and stimulated conditions (Figure 5E and ref. 6), and such an effect can be directly attributed to coexpression of WT proinsulin (Figure 3B). Moreover, rescue of mutant proinsulin- $\mathrm{G}(\mathrm{B} 23) \mathrm{V}$ or $r d w$-Tg is also protein specific: in cells coexpressing both mutants, secretory rescue of 


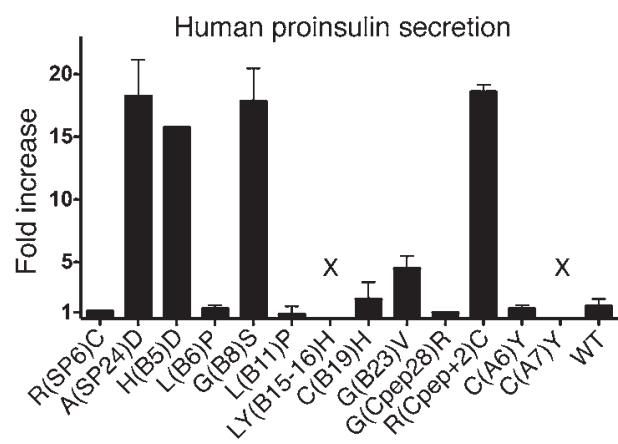

\section{Figure 8}

Secretory rescue by WT proinsulin is restricted to a subset of MIDY mutants. 293T cells transiently cotransfected with plasmids expressing the indicated hPro mutants and either empty vector or WT mPro were incubated overnight in growth medium beginning at 24 hours after transfection. Media were collected, and hPro secretion was measured by RIA. The data shown (fold increase in mutant proinsulin secretion as a consequence of expressing WT mPro over empty vector) are mean values \pm range from 2 independent experiments. $X$, undetectable.

proinsulin-G(B23)V was accomplished exclusively by WT proinsulin, whereas secretory rescue of $r d w$-Tg was accomplished exclusively by WT Tg (Figure 4).

All evidence points to the idea that secretory rescue is a consequence of intracellular stabilization of the mutant gene product: this was true for both proinsulin-G(B23)V (Figure 5A) and $r d w-\mathrm{Tg}$ (Figure 5B). And for both mutant proteins, the magnitude of the stabilization and secretory rescue is linked to the ratio of WT/ mutant protein expression (Figure 5, C and D). Most remarkable of all, rescue (of mutant) and blockade (of WT) secretion occurred simultaneously in the same cells (Figure 7). Thus, for both proteins, there is a dynamic bidirectional balance between retention and anterograde transport of mutant cross-dimers (Figure 9).

We posit that, for the many conformational diseases affecting exportable proteins that oligomerize in the $\mathrm{ER}$, a dominant versus recessive pattern of inheritance is in part a reflection of the balance of these two activities (blockade versus rescue; see Figure 9). In the case of MIDY and other dominantly inherited diseases, the balance favors net retention of protein, whereas, in the case of congenital hypothyroidism with defective $\mathrm{Tg}$, the balance favors net export. The minimum plasmid ratio at which we observed secretory rescue was consistent with this hypothesis: rescue of proinsulin- $\mathrm{B} 23 \mathrm{~V}$ required higher $\mathrm{WT} /$ mutant ratios than the rescue of $r d w$-Tg secretion (Figure 5).

In conclusion, the data presented herein indicate that both secretory blockade and rescue involve direct cross-dimerization between WT and mutant gene products. As cross-dimerization in the secretory pathway is certainly not limited to proinsulin and $\operatorname{Tg}(55)$, we expect that similar cooperativity will be observed for other exportable proteins. While the effects described in this report appear limited to protein-specific protein rescue of mutant oligomerization partners, the results imply a broader significance for understanding disease pathophysiology. Specifically, our studies have potential relevance for the finding of ER accumulation of secretory proteins, even in the absence of any mutations. For example, WT proinsulin is prone to misfolding under conditions of increased insulin demand $(56,57)$, and its accumulation may contribute to $\beta$ cell failure $(58,59)$. If oligomerization plays an impor- tant role in the retention of WT proinsulin and other WT secretory proteins, then protecting/stabilizing the interaction interface (such as with small molecule interactors) might allow ER escape of an increased fraction of exportable protein, despite the presence of a misfolded subset of such molecules. Such methods might be used in combination therapy with other approaches to alter the ER environment (described above), opening remarkable new avenues for treatment of diseases of misfolding of exportable proteins.

\section{Methods}

Materials. Lipofectamine 2000, DMEM, RPMI 1640 medium, fetal bovine serum, Zysorbin, penicillin, and streptomycin were from Invitrogen. Glucose, 3-isobutyl-1-methylxanthine (IBMX), tolbutamide, and brefeldin A were from Sigma-Aldrich. EndoH was from New England Biolabs. Complete protease inhibitor cocktail was from Roche. Citrisolv was from Fisher Scientific. Rabbit anti-Myc and anti-GFP and chicken anti-Myc were from Immunology Consultants. Mouse mAb anti-HA was from Covance. Rabbit anti-ACTH antibody was a gift from M. Low (University of Michigan). hPro-specific RIA was from Millipore. mPro-specific ELISA and mPro-specific antibody was from Alpco. Trans ${ }^{35} \mathrm{~S}$ label and $\mathrm{Na}^{125} \mathrm{I}$ were from Perkin Elmer.

Cell culture and transfection. 293, 293T, and AtT20 cells were cultured in DMEM with $10 \%$ fetal bovine serum and penicillin $(100 \mathrm{U} / \mathrm{ml})$ and streptomycin $(100 \mu \mathrm{g} / \mathrm{ml})$. INS1 and INS1E cells were cultured in RPMI 1640 medium supplemented with $10 \%$ fetal bovine serum, $1 \mathrm{mM}$ pyruvate, $10 \mathrm{mM}$ HEPES, penicillin $(100 \mathrm{U} / \mathrm{ml})$, streptomycin $(100 \mu \mathrm{g} / \mathrm{ml})$, and $50 \mathrm{mM}$ 2 -mercaptoethanol. Proinsulin and Tg variants were expressed in

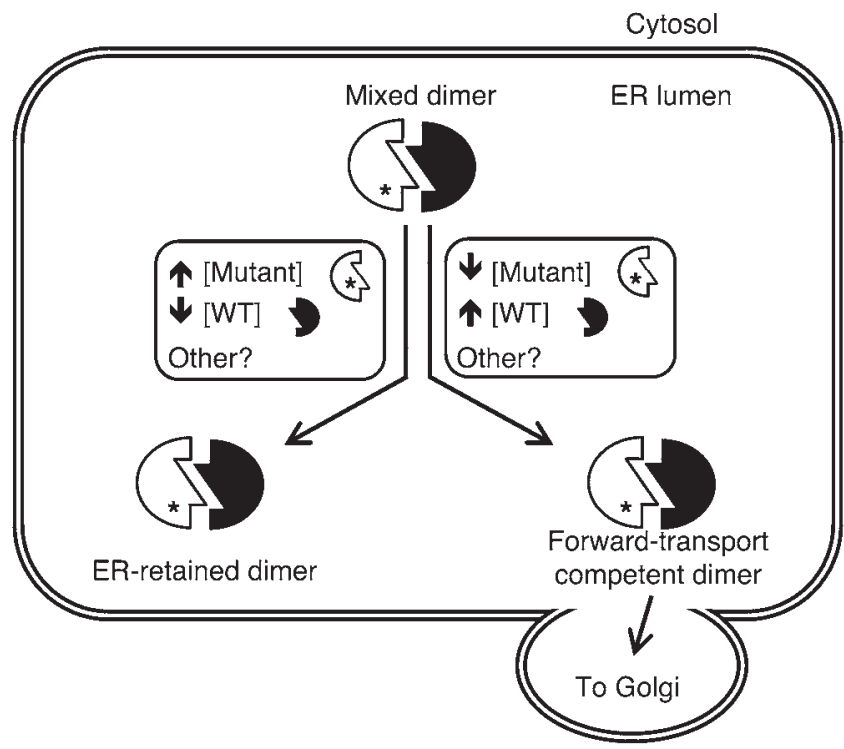

\section{Figure 9}

Model of bidirectional intermolecular interactions of misfolded and native proteins. Within the ER, many secretory proteins, including proinsulin and Tg, form homodimers. When mutant and WT alleles from the same gene cross-dimerize, there may be several outcomes, two of which are summarized in the figure. The WT gene product can assist the mutant partner to exit the ER, or the mutant protein can block anterograde transport of the WT protein. Among other protein-specific and general factors involved, the relative concentrations (i.e., stoichiometric ratio) of the 2 dimerization partners also contribute to the outcome, with lower WT/mutant ratios resulting in greater ER retention and higher WT/mutant ratios resulting in enhanced forward transport. 
pCDNA3.1 or pTarget mammalian expression vectors. The hPro-CpepSfGFP-KDEL vector was a gift from E. Snapp (AECOM). Transfections using Lipofectamine 2000 were performed in 12 -well plates. Total plasmid DNA was held constant within each experiment by addition of empty vector. Cells were harvested 24-48 hours after transfection and lysed in boiling SDS gel buffer (4\% SDS, 20\% glycerol, $120 \mathrm{mM}$ Tris, pH 6.8), RIPA buffer (0.1 M NaCl, $0.2 \%$ deoxycholate, $25 \mathrm{mM}$ Tris, $\mathrm{pH} 7.4,1 \%$ Triton X-100, $0.1 \%$ SDS, 10 mM EDTA, pH 8.0, and proteinase inhibitor cocktail), NP40CoIP buffer (1\% NP40, 0.1 M NaCl, 2 mM EDTA, 25 mM Tris, pH 7.4), or TX-CoIP buffer (0.1\% Triton X-100, $0.1 \mathrm{M} \mathrm{NaCl}, 5 \mathrm{mM}$ EDTA, $25 \mathrm{mM}$ Tris, $\mathrm{pH} 7$ ), as indicated. For glucose-stimulated secretion measurements, cells were preincubated in $2.8 \mathrm{mM}$ glucose for 30 minutes. Fresh basal media (2.8 mM glucose) was then collected for 90 minutes, followed by stimulation media (21 mM glucose, $1 \mathrm{mM}$ tolbutamide, $1 \mathrm{mM}$ IBMX) for 90 minutes, with cell lysis thereafter in SDS gel buffer.

Generation of mouse lines. Mice expressing hProC(A7)Y-CpepGFP transgene driven by the Ins 1 promoter, bearing heterozygous/homozygous disruption of endogenous Ins2, were as previously described (22). A full description of $r d w$-Tg3xMyc transgenic mice is forthcoming; briefly, a transgene consisting of the bovine Tgn promoter (60) immediately upstream of the full-length mouse $\operatorname{Tgn}$ ORF encoding $r d w$-Tg plus a tripleMyc epitope tag (i.e., $r d w$-Tg3xMyc) (10) was expressed in C57BL/6 mice. These $r d w$-Tg3xMyc transgenic mice in a $\operatorname{Tg}^{+/+}$genetic background were crossed and then backcrossed with $\operatorname{Tg} n^{\operatorname{cog} / \operatorname{cog}}$ mice to generate $\operatorname{Tg} n^{+/ \operatorname{cog}}$ mice and then $\operatorname{Tg} n^{\operatorname{cog} / \operatorname{cog}}$ mice with or without the $r d w$-Tg3xMyc transgene. All animals were used in accordance with the University of Michigan's University Committee on Use and Care of Animals.

Confocal imaging. Formaldehyde-fixed cells were permeabilized with $0.4 \%$ TX100, blocked (TBS containing 3\% BSA and 0.2\% TX100), and then either directly mounted or stained overnight at $4{ }^{\circ} \mathrm{C}$ with primary antibodies: chicken anti-Myc (1:5,000 dilution) and rabbit anti-ACTH $(1: 25,000)$. Thereafter, slides were rinsed and incubated with secondary antibody conjugates, mounted with ProLong Gold with DAPI (Invitrogen), and imaged by confocal epifluorescence with a $\times 60$ oil objective. GFP intensity in granule (ACTH staining) and nongranule regions was quantified using MetaMorph (Molecular Devices). For cells transfected with hPro-CpepMyc, only cells positively staining with chicken anti-Myc antibodies were quantified.

For imaging of mouse islets, excised pancreata were fixed, paraffin embedded, microsectioned, and deparaffinized with Citrisolv. Sections underwent antigen retrieval (RetrieveALL-1), were blocked with 3\% BSA, and were immunostained overnight at $4{ }^{\circ} \mathrm{C}$ with mouse anti-mPro, guinea pig anti-insulin, and rabbit anti-calnexin antibodies. After rinsing and incubation with secondary antibody conjugates, slides were mounted with ProLong Gold with DAPI and by confocal epifluorescence as above. For quantification of WT mPro localization, investigators were blinded to mouse genotype. Four to five islets per mouse were analyzed, with each nucleated cell expressing proinsulin scored as either "majority ER" (colocalizing with calnexin) or "majority Golgi" (perinuclear crescent of increased signal intensity).

Metabolic labeling, immunoprecipitation, and EndoH digestion. Twenty-four hours after transfection, cells were trypsinized and replated on poly-Dlysine-coated plates. After 6 hours, cells were pulse labeled with ${ }^{35} \mathrm{~S}$-amino acids for 10 minutes and chased as indicated with cells lysed in RIPA buffer. For measurement of mutant proinsulin stability, lysate and media were combined, precleared with zysorbin, and immunoprecipitated with anti-insulin or anti-Myc. Immunoprecipitates (normalized to TCA-precipi- table counts) were analyzed by reducing $4 \%-12 \%$ acrylamide gradient SDSPAGE, phosphorimaging, and band quantitation (ImageQuant).

For ${ }^{125} \mathrm{I}$ labeling of secreted $r d w$-Tg3xMyc, thyroid glands were labeled for 30 minutes at $37^{\circ} \mathrm{C}$ in $200 \mu \mathrm{l}$ complete medium containing $0.1 \mu \mathrm{M} \mathrm{NaI}$ plus $1.0 \mu \mathrm{Ci} / \mu \mathrm{l} \mathrm{Na}{ }^{125} \mathrm{I}$. After labeling, samples were washed thrice with ice-cold PBS, sonicated in $200 \mu \mathrm{l}$ RIPA buffer, diluted to $1 \mathrm{ml}$, and immunoprecipitated with rabbit anti-Myc antibody normalized to total DNA in the lysate.

EndoH digestion was performed according to manufacturer instructions; EndoH-sensitive and -resistant $r d w$-Tg3xMyc standards were from transfected $293 \mathrm{~T}$ cells radiolabeled with ${ }^{35} \mathrm{~S}$-amino acids. EndoH digests were analyzed by SDS 5\%-PAGE and phosphorimaging. Controls establish that anti-Myc does not immunoprecipitate untagged endogenous $\operatorname{Tg}$ (data not shown).

Coimmunoprecipitation. After lysis in ice-cold coimmunoprecipitation (CoIP) buffer, lysates were precleared and then immunoprecipitated with the indicated antibodies, washed, and analyzed by reducing SDSPAGE and immunoblotting.

Proinsulin measurements and Western blotting. At 24 hours after transfection, an overnight incubation in fresh media was initiated, and cells were lysed in RIPA buffer. Secreted and cellular proinsulin was measured by hPro-specific RIA or mPro-specific ELISA normalized to total protein in the lysate. For Western blotting, proteins ( $10 \mu \mathrm{g}$ per lane) were resolved by $4 \%-12 \%$ acrylamide gradient SDS-PAGE, electrotransferred to nitrocellulose, and immunoblotted with the indicated antibodies. Horseradish peroxidaseconjugated secondary antibodies were from Jackson ImmunoResearch, with proteins visualized by ECL (Millipore). $\alpha$-Tubulin was measured as a loading control. Western blot bands were quantified using ImageJ and ImageQuant software. The location of relevant molecular weight markers $(\mathrm{kDa})$ is indicated in the figures.

Statistics. Statistical analyses were conducted using GraphPad Prism software. Data are presented as mean \pm SEM, unless otherwise noted (as in the legend for Figure 8). Two-tailed Student's $t$ test was used to assess statistical significance, with a threshold for significance of $P<0.05$.

Study approval. Handling and euthanizing of mice were performed entirely in accordance with national guidelines and with approval from the Committee on Use and Care of Animals at the University of Michigan.

\section{Acknowledgments}

This work was supported by NIH R01DK48280 and R01DK40344 with additional support from March of Dies grant no. 6-FY11357 as well as support from training grants NIH F30DK095504, T32GM007863, T32GM008322, and T32HD007505. The authors thank Stephen Lentz at the Morphology and Image Analysis Core and the Transgenic Animal Model Core, both supported by the Michigan Diabetes Research Center (NIH P30DK020572) and Michigan Gastrointestinal Peptide Research Center (NIH P30DK034933). We thank Ann Soliman, Ali Reda, and Kathryn Hutchison for technical assistance.

Received for publication October 8, 2012, and accepted in revised form March 28, 2013.

Address correspondence to: Peter Arvan, Division of Metabolism, Endocrinology and Diabetes, University of Michigan, Brehm Tower Rm 5112, 1000 Wall St., Ann Arbor, Michigan 48105, USA. Phone: 734.936.5505; Fax: 734.936.6684; E-mail: parvan@umich.edu.
1. Aridor M, Hannan LA. Traffic jam: a compendium of human diseases that affect intracellular transport processes. Traffic. 2000;1(11):836-851.

2. Kim PS, Arvan P. Endocrinopathies in the family of endoplasmic reticulum (ER) storage diseases: disorders of protein trafficking and the role of ER molecular chaperones. Endocr Rev. 1998;19(2):173-202.

3. Dodson G, Steiner D. The role of assembly in insulin's biosynthesis. Curr Opin Struct Biol. 1998; 8(2):189-194.

4. Liu M, et al. Proinsulin misfolding and diabetes: mutant INS gene-induced diabetes of youth. Trends 
Endocrinol Metab. 2010;21(11):652-659.

5. Liu M, et al. Mutant INS-gene induced diabetes of youth: proinsulin cysteine residues impose dominant-negative inhibition on wild-type proinsulin transport. PLoS One. 2010;5(10):e13333.

6 . Rajan $S$, et al. In vitro processing and secretion of mutant insulin proteins that cause permanent neonatal diabetes. Am J Physiol Endocrinol Metab. 2010; 298(3):E403-E410.

7. Arvan P, Di Jeso B. Thyroglobulin structure, function, and biosynthesis. In: Braverman LE, Utiger R, eds. The Thyroid. Philadelphia, Pennsylvania, USA: Lippincott Williams and Wilkins; 2004:77-95.

8. Kim PS, Arvan P. Folding and assembly of newly synthesized thyroglobulin occurs in a pre-Golgi compartment. J Biol Chem. 1991;266(19):12412-12418.

9. Lee J, Di Jeso B, Arvan P. The cholinesterase-like domain of thyrogobulin functions as an intramolecular chaperone. J Clin Invest. 2008;118(8):2950-2958.

10. Lee J, Wang X, Di Jeso B, Arvan P. The cholinesterase-like domain, essential in thyroglobulin trafficking for thyroid hormone synthesis, is required for protein dimerization.J Biol Chem. 2009; 284(19):12752-12761.

11. Targovnik HM, Esperante SA, Rivolta CM. Genetics and phenomics of hypothyroidism and goiter due to thyroglobulin mutations. Mol Cell Endocrinol. 2010;322(1-2):44-55.

12. Kim PS, Hossain SA, Park YN, Lee I, Yoo SE, Arvan $P$. A single amino acid change in the acetylcholinesterase-like domain of thyroglobulin causes congenital goiter with hypothyroidism in the cog/ cog mouse: a model of human endoplasmic reticulum storage diseases. Proc Natl Acad Sci U S A. 1998; 95(17):9909-9913.

13. Hishinuma A, Furudate S, Oh-Ishi M, Nagakubo $\mathrm{N}$, Namatame T, Ieiri T. A novel missense mutation (G2320R) in thyroglobulin causes hypothyroidism in rdw rats. Endocrinology. 2000;141(11):4050-4055.

14. Kim PS, et al. A missense mutation G2320R in the thyroglobulin gene causes non-goitrous congenital primary hypothyroidism in the WIC-rdw rat. $\mathrm{Mol}$ Endocrinol. 2000;14(12):1944-1953.

15. Umezu M, Kagabu S, Jiang J, Sato E. Evaluation and characterization of congenital hypothyroidism in rdw dwarf rats. Lab Anim Sci. 1998;48(5):496-501.

16. Menon S, et al. Oxidoreductase interactions include a role for ERp72 engagement with mutant thyroglobulin from the rdw/rdw rat dwarf. J Biol Chem. 2007;282(9):6183-6191.

17. Wang $X$, et al. Cis and trans actions of the cholinesterase-like domain within the thyroglobulin dimer. J Biol Chem. 2010;285(23):17564-17573.

18. Hodish I, et al. Misfolded proinsulin affects bystander proinsulin in neonatal diabetes. J Biol Chem. 2010;285(1):685-694

19. Gupta S, McGrath B, Cavener DR. PERK (EIF2AK3) regulates proinsulin trafficking and quality control in the secretory pathway. Diabetes. 2010; 59(8):1937-1947.

20. Haataja L, et al. Proinsulin intermolecular interactions during secretory trafficking in pancreatic $\beta$ cells. J Biol Chem. 2013;288(3):1896-1906.

21. Fogelfeld L, Harel G, Beamer WG, Schneider AB. Low-molecular-weight iodoproteins in the congenital goiters of $\mathrm{cog} / \operatorname{cog}$ mice. Thyroid. 1992; 2(4):329-335.

22. Hodish I, et al. In vivo misfolding of proinsulin below the threshold of frank diabetes. Diabetes. 2011; 60(8):2092-2101.

23. Orci L, Ravazzola M, Amherdt M, Madsen O, Vassalli JD. Direct identification of prohormone conversion site in insulin-secreting cells. Cell. 1985; 42(2):671-681.
24. Schuit FC, In't Veld PA, Pipeleers DG. Glucose stimulates proinsulin biosynthesis by a dose-dependent recruitment of pancreatic $\beta$ cells. Proc Natl Acad Sci U S A. 1988;85(11):3865-3869.

25. Kiekens R, In 't Veld P, Mahler T, Schuit F, Van De Winkel M, Pipeleers D. Differences in glucose recognition by individual rat pancreatic $B$ cells are associated with intercellular differences in glucose-induced biosynthetic activity. J Clin Invest. 1992; 89(1):117-125.

26. Nehls S, et al. Dynamics and retention of misfolded proteins in native ER membranes. Nat Cell Biol. 2000; 2(5):288-295

27. Guerriero CJ, Brodsky JL. The delicate balance between secreted protein folding and endoplasmic reticulum-associated degradation in human physiology. Physiol Rev. 2012;92(2):537-576.

28. Hulleman JD, Balch WE, Kelly JW. Translational attenuation differentially alters the fate of disease-associated fibulin proteins. FASEB J. 2012; 26(11):4548-4560

29. Yu T, Chung C, Shen D, Xu H, Lieberman AP. Ryanodine receptor antagonists adapt NPC1 proteostasis to ameliorate lipid storage in Niemann-Pick type C disease fibroblasts. Hum Mol Genet. 2012;21(14):3205-3214

30. Wang F, Chou A, Segatori L. Lacidipine remodels protein folding and Ca 2+ homeostasis in Gaucher's disease fibroblasts: a mechanism to rescue mutant glucocerebrosidase. Chem Biol. 2011; 18(6):766-776

31. Yu T, Chung C, Shen D, Xu H, Lieberman AP. Ryanodine receptor antagonists adapt NPC1 proteostasis to ameliorate lipid storage in Niemann-Pick type C disease fibroblasts. Hum Mol Genet. 2012;21(14):3205-3214

32. Conn PM, Janovick JA. Drug development and the cellular quality control system. Trends Pharmacol Sci. 2009;30(5):228-233.

33. Balch WE, Morimoto RI, Dillin A, Kelly JW. Adapting proteostasis for disease intervention. Science. 2008;319(5865):916-919.

34. Lukacs GL, Verkman AS. CFTR: folding, misfolding and correcting the $\Delta \mathrm{F} 508$ conformational defect. Trends Mol Med. 2012;18(2):81-91.

35. Lu PD, et al. Cytoprotection by pre-emptive conditional phosphorylation of translation initiation factor 2. EMBO J. 2004;23(1):169-179.

36. Hurtley SM, Helenius A. Protein oligomerization in the endoplasmic reticulum. Annu Rev Cell Biol. 1989; 5:277-307

37. Bole DG, Hendershot LM, Kearney JF. Posttranslational association of immunoglobulin heavy chain binding protein with nascent heavy chains in nonsecreting hybridomas. J Cell Biol. 1986; 102(5):1558-1566

38. Doms RW. Oligomerization and protein transport. Methods Enzymol. 1990;191:841-854.

39. Leitzgen K, Knittler MR, Haas IG. Assembly of immunoglobulin light chains as a prerequisite for secretion. A model for oligomerization-dependent subunit folding. J Biol Chem. 1997;272(5):3117-3123.

40. Kerem A, Kronman C, Bar-Nun S, Shafferman A, Velan B. Interrelations between assembly and secretion of recombinant human acetylcholinesterase. J Biol Chem. 1993;268(1):180-184.

41. Reddy PS, Corley RB. Assembly, sorting, and exit of oligomeric proteins from the endoplasmic reticulum. Bioessays. 1998;20(7):546-554.

42. Anelli T, et al. Thiol-mediated protein retention in the endoplasmic reticulum: the role of ERp44. EMBO J. 2003;22(19):5015-5022.

43. Kim P, Bole D, Arvan P. Transient aggregation of nascent thyroglobulin in the endoplasmic reticu- lum: relationship to the molecular chaperone, BiP. J Cell Biol. 1992;118(3):541-549.

44. Lodish HF, Kong N, Wikstrom L. Calcium is required for folding of newly made subunits of the asialoglycoprotein receptor within the endoplasmic reticulum. J Biol Chem. 1992;267(18):12753-12760.

45. De Jaco A, et al. A mutation linked with autism reveals a common mechanism of endoplasmic reticulum retention for the alpha,beta-hydrolase fold protein family. J Biol Chem. 2006;281(14):9667-9676.

46. Hahn MK, Robertson D, Blakely RD. A mutation in the human norepinephrine transporter gene (SLC6A2) associated with orthostatic intolerance disrupts surface expression of mutant and wild-type transporters. J Neurosci. 2003;23(11):4470-4478.

47. Cordat E, et al. Dominant and recessive distal renal tubular acidosis mutations of kidney anion exchanger 1 induce distinct trafficking defects in MDCK cells. Traffic. 2006;7(2):117-128.

48. Mendes HF, Cheetham ME. Pharmacological manipulation of gain-of-function and dominant-negative mechanisms in rhodopsin retinitis pigmentosa. Hum Mol Genet. 2008;17(19):3043-3054.

49. Kagan A, Yu Z, Fishman GI, McDonald TV. The dominant negative LQT2 mutation A561V reduces wild-type HERG expression. J Biol Chem. 2000; 275(15):11241-11248

50. Butler DC, McLear JA, Messer A. Engineered antibody therapies to counteract mutant huntingtin and related toxic intracellular proteins. Prog Neurobiol. 2012;97(2):190-204.

51. Li C, Xiao P, Gray SJ, Weinberg MS, Samulski RJ. Combination therapy utilizing shRNA knockdown and an optimized resistant transgene for rescue of diseases caused by misfolded proteins. Proc Natl Acad SciU S A. 2011;108(34):14258-14263.

52. Sandefur CI, Schnell S. A model of threshold behavior reveals rescue mechanisms of bystander proteins in conformational diseases. Biophys J. 2011; 100(8):1864-1873.

53. Navarro D, Qadri I, Pereira L. A mutation in the ectodomain of herpes simplex virus 1 glycoprotein $B$ causes defective processing and retention in the endoplasmic reticulum. Virology. 1991; 184(1):253-264.

54. Marquardt T, Helenius A. Misfolding and aggregation of newly synthesized proteins in the endoplasmic reticulum. J Cell Biol. 1992;117(3):505-513.

55. Kittanakom S, Cordat E, Akkarapatumwong V, Yenchitsomanus PT, Reithmeier RA. Trafficking defects of a novel autosomal recessive distal renal tubular acidosis mutant (S773P) of the human kidney anion exchanger (kAE1). J Biol Chem. 2004; 279(39):40960-40971

56. Wang J, Chen Y, Yuan Q, Tang W, Zhang X, Osei K. Control of precursor maturation and disposal is an early regulative mechanism in the normal insulin production of pancreatic beta-cells. PLoS One. 2011; 6(4):e19446.

57. Liu M, Li Y, Cavener D, Arvan P. Proinsulin disulfide maturation and misfolding in the endoplasmic reticulum. J Biol Chem. 2005;280(14):13209-13212.

58. Laybutt DR, et al. Endoplasmic reticulum stress contributes to beta cell apoptosis in type 2 diabetes. Diabetologia. 2007;50(4):752-763.

59. Scheuner D, Kaufman RJ. The unfolded protein response: a pathway that links insulin demand with beta-cell failure and diabetes. Endocr Rev. 2008; 29(3):317-333.

60. Ledent C, Parmentier M, Vassart G. Tissue-specific expression and methylation of a thyroglobulin-chloramphenicol acetyltransferase fusion gene in transgenic mice. Proc Natl Acad Sci U S A. 1990; 87(16):6176-6180. 